\title{
Observations sur l'évolution des grottes ${ }^{1}$ )
}

\author{
Par Albert Cavaillét 2 ) \\ Asec $\%$ figures dans le texte
}

\section{Le système karstique élémentaire}

Après les travaux de Cvijic (1918) précisés par O.Lehmann (1932), J. Kunsky (1950) et plus récemment par B. Gèze et Ph. Renault (1962) nous avons une image assez nette des circulations souterraines sous les plateaux calcaires de constitution homogène. Les exemples abondent en France de ces structures tabulaires: Grands Causses et Causses du Périgord et du Quercy, plans de Provence, plateaux de Garrigues de l'Hérault et du Gard, plateaux de Bourgogne. L'histoire morphologique est certes différente dans chaque cas, mais dans leur état actuel, et quelle que soit l'altitude relative de ces plateaux par rapport aux vallées qui les bordent, la circulation souterraine qui les caractérise peut ètre décrite selon un schéma voisin de celui que nous avons déjà exposé, en raisonnant sur un exemple, en 1950.

- A la base, c'est-à-dire au-dessous du niveau des rivières qui bordent les plateaux, un réseau de galeries noyées se vide par les sources vauclusiennes pérennes: nappe en réseau, réseau de fentes.

- Au-dessus de cette zone noyée, un niveau de galeries alternativement libres ou pleines d'eau s'individualise en arrière des sources intermittentes, des trop-pleins, des porches de grottes inférieuses.

- L'alimentation de ces niveaux inférieurs est assurée par des chenaux verticaux qui concentrent plus ou moins les eaux infiltrées à la surface du plateau.

- Dans les parties superficielles, des galeries étroites, prolongeant parfois les formes karstiques de surface (lapiaz) convergent vers ces galeries verticales qui, à l'origine, ne s'ouvrent pas à l'air libre par des passages explorables.

La forme primitive des galeries dépend beaucoup de la lithologie. Toutes les retouches ultérieures qui modifient des formes primitives

1) Le présent travail est la mise à jour d'une étude présentée au Congrès National de Belfort-Malvaux en 1962 - A. Cavaillé, professeur, Ecole Normale de Montauban (T. et G.)

2 ) Président de la Fédération Française de Spéléologie, chemin du Coteau, Montauban (T. et G.) 
jusqu'aux aspects qu'observent les spéléologues sont des détails, qu'il est parfois difficile de distinguer des phénomènes primordiaux.

$a$ - Les conduits de surface

Leur observation directe est impossible; on ne les connait qu'indirectement, par exemple par l'étude minutieuse des lapiaz. Leurs canelures conduisent l'eau jusqu'à des diaclases. Celles-ci, mème dans les cas les plus favorables, échappent à l'observation. Parfois, les carrières ou les tranchées les découvrent: elles ont une largeur de l'ordre du centimètre au décimètre, et ne sont que rarement béantes.

En effet, les eaux qui imbibent le sol résiduel sont très vite saturées de carbonate de chaux et l'argile flocule sur place; d'autre part, aucun courant ne peut exercer d'action érosive, ni de transport d'éléments solides. La profondeur des diaclases corrodées plus largement dépend de la roche et du climat; ce sont les facteurs qui favorisent la formation des lapiaz: calcaire pur et climats nivaux. Dans les situations actuelles des bas-plateaux peu enneigés, même dans des conditions favorables de la lithologie (par exemple sur les Causses mineurs ou les plateaux de Bourgogne) la surface s'use uniformément sous le manteau du sol résiduel, actuel ou fossile, et l'infiltration des eaux est immédiate; le réseau des fentes est très dense et les diaclases très peu élargies. Sur les plateaux plus élevés (Vercors, hautes surfaces calcaires des Pyrénées), les lapiaz sont plus ou moins amples, mais les diaclases qui les poursuivent vers le bas sont très généralement inexplorables.

Ce n'est que dans les cas où l'action mécanique du gel-degel s'exerce sur une roche favorable comme la dolomie, que des éclatements et des éboulements peuvent se produire; on a alors les puits à neige, toujours peu profonds, comme ceux que nous avons pu observer sur le Dachstein autrichien ou les «scialets» décrits par le groupe des Cyclopes sur le Massif de Presles (Vercors).

\section{$b$ - Les galeries verticales du système karstique}

Les eaux infiltrées de la surface sont donc très dispersées; elles se glissent dans les diaclases, s'insinuent entre les joints, tout en poursuivant leur travail de corrosion, réduit par la faiblesse de leurs débits.

Ces filets d'eau finissent par se concentrer vers une diaclase plus importance ou sont guidées par le carrefour de plusieurs cassures.

Leur force de corrosion peut alors augmenter, surtout à la fonte des neiges, ou après les fortes pluies, lorsque la traversées de la zone superficielle est rapide. La turbulence de ces petits courants accroit leur possibilité d'action corrosive. Tous les phénomènes chimiques, dans cette atmosphère peu renouvelée, certainement riche en gaz carbonique, 


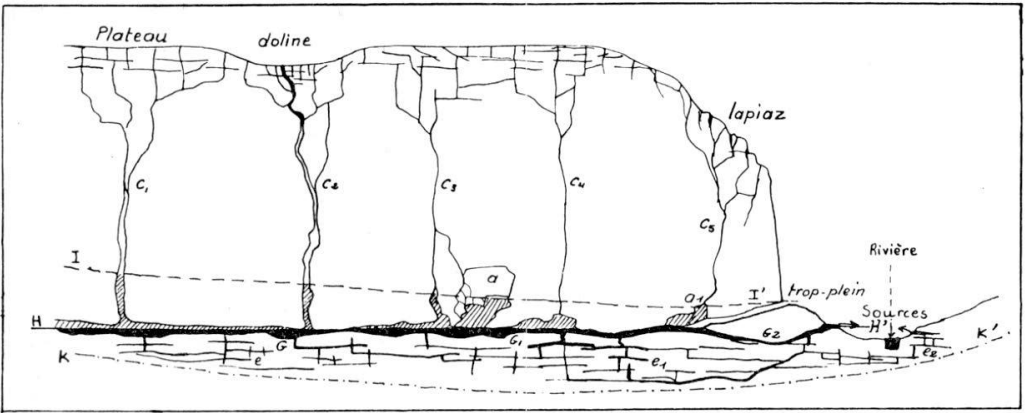

Fig. 1: Schéma du système karstique. Circulation actuelle de l'eau dans une masse calcaire homogène. En surface, diaclases et joints absorbants; $\mathrm{c}-\mathrm{c}_{5}$, puits de descente rapide des eaux; $\mathrm{G}-\mathrm{G}_{2}$, galeries horizontales des circulations karstiques avec salles d'éboulis a ; $\mathrm{e}-\mathrm{e}_{2}$ diaclases et joints du réseau de fentes. $-\mathrm{K}^{\prime} \mathrm{K}^{\prime}=$ niveau de base karstique, $\mathrm{H}^{\prime} \mathrm{H}^{\prime}=$ niveau piézométrique d'étiage, I I' = niveau de hautes eaux.

se fait selon le processus élémentaire; la diaclase primitive s'élargit, des tourbillons s'amorcent, et ainsi s'ouvrent les "cheminées" qui s'observent par le bas à la voûte des galeries, le plus souvent en section circulaire, parfois de forme hélicoïdale.

Le diamètre de ces galeries de descente croît vers le bas, avec la concentration des eaux du plateau, et aussi avec la vitesse du courant qui la corrode. Mais il reste généralement petit, de l'ordre du décimètre au mètre.

Pour une même formation lithologique, ce diamètre dépend de la profondeur de calcaire traversé: plus elle est grande, plus la concentration du drainage est importante. C'est bien ce que semble montrer l'observation; sous les bas plateaux, les cheminées au plafond des galeries horizontales sont petites et peuvent passer inaperçues (par exemple à la grotte de Miramont); sous les plateaux élevés, il peut s'ouvrir des cheminées (qui donneront des gouffres) de gros diamètre.

\section{$c$ - Les galeries noyées}

Ce sont celles qui se trouvent sous le niveau des plus basses résurgences. A l'étiage, lorsque l'ensemble tend vers l'équilibre, l'eau remplit les joints et les diaclases au-dessous du niveau horizontal marqué par le niveau de la plus basse résurgence régionale. Ce niveau hydrostatique n'est pas le niveau de base karstique; en effet, le creusement de la roche, l'élargissement des joints et des diaclases se poursuit bien au-dessous de ce plan horizontal, jusqu'à des profondeurs de l'ordre de plusieurs décamètres. 
L'existence de ces galeries noyées, longtemps ignorée ou niée par principe, est maintenant admise comme un phénomène général; les plongées ont permis de les reconnaitre, les colorations nombreuses ont servi à évaluer leur volume total et même leur agencement. Nous savons qu'elles sont plus ou moins indépendantes des rivières aériennes; ainsi s'expliquent par exemple, les ressorties de fluorescéïne sur les deux rives d'une rivière, après une coloration faite à l'amont (de Lavaur, A. Cavaillé).

La forme de ces galeries noyées semble assez diverse; ce sont des joints ou des diaclases élargies en longues fentes irrégulières, de position variable selon le pendage. Les bouches des résurgences en forment un cas particulier: en effet, ici encore, la concentration du drainage souterrain provoque un fort débit dans un chenal privilégié, et la turbulence augmente la corrosion; dans certaines résurgences de type «fourfoul» le frottement contre les parois des cailloux venus de l'extérieur et qui s'arrondissent au cours de leurs perpétuels voyages de bas en haut et de haut en bas, peut entraîner la formation de stries ou de cannelures plus ou moins hélicoïdales sur la bouche de la source. De nombreuses ouvertures de grottes, à Lascaux par exemple, montrent ces sculptures faites par le courant de résurgence.

Mais, excepté ce cas, toutes les galeries, sous le niveau hydrostatique, s'ouvrent par corrosion. Et, dans ces chenaux élémentaires, les conditions de la dissolution sont extrêmement variables. Elles dépendent d'abord de la nature de la roche souvent hétérogène; ainsi s'expliquent les poches, le fentes, le hérissement en lames ou en tubercules dû aux zones moins solubles de la masse calcaire. Lorsque l'exploration des galeries conduit jusqu'à ces zones basses, on voit les traces de cette corrosion différentielle, qui aboutit à la formation de passages extrêmement irréguliers, comme une véritable dentelle ouverte dans les strates ou contournant les blocs (A. Cavaillé, 1963).

Dans cette zone noyée du système karstique, la corrosion se fait le plus souvent en eau parfaitement tranquille; les courants y sont extrêmement lents, surtout dans les parties les plus profondes; en principe, il n'y a pas de remplissage: seul le résidu de la dissolution peut s'accumuler sur place, combler quelque diaclase, colmater quelque joint, mais la dissolution s'exerce à côté du placage argileux qui reste toujours peu important.

Les auteurs appellent souvent ces chenaux constamment noyés: "galeries en conduite forcée». En fait ce terme est très impropre; l'eau y est en effet en équilibre; elle ne s'y renouvelle que très lentement, ce qui permet cependant à la corrosion de s'y exercer constam- 
ment, quoique avec très peu d'intensité; les courants qui les affectent sont localisés, lors des mises en charges du niveau supérieur au moment des pluies, dans la zone d'alimentation du système karstique ou par effet de trompe du côté de la résurgence. En définitive, les diaclases primitives évoluent peu à peu, et les galeries tendent à se régulariser: elles deviennent le plus souvent, dans un calcaire compact homogène, des tunnels à section circulaire, dont le diamètre est proportionnel aux débits.

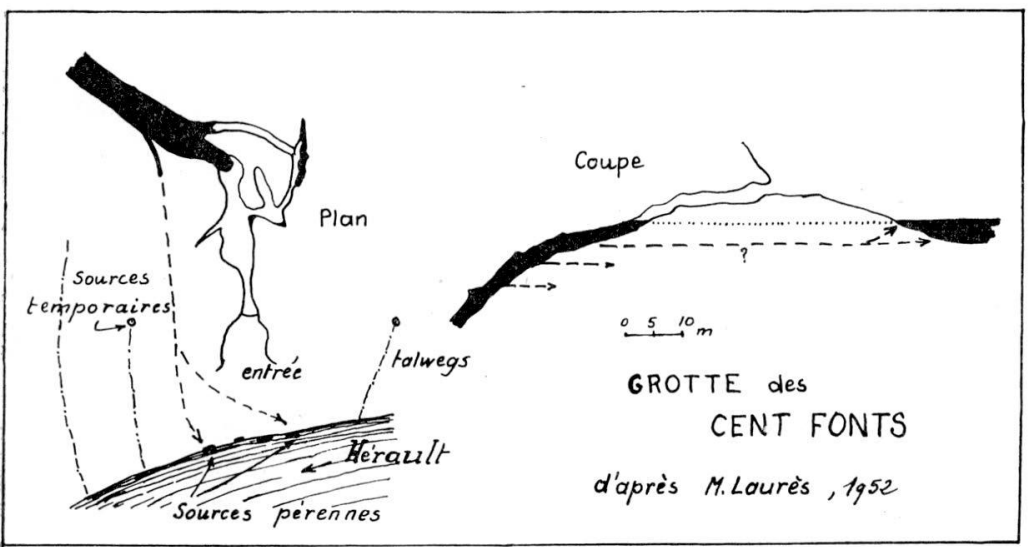

Fig. 2: Grotte des Cent Fonts; elle montre la présence de galeries embryonnaires au dessous du niveau de l'Hérault. Lorsque celui-ci aura approfondi son lit, les sources pérennes de ses berges deviendront les exutoires du système karstique.

Echappant le plus souvent à l'exploration, la zone noyée du système karstique n'en a pas moins une grande importance au point de vue hydraulique. Les colorations à la fluoresceïne ont permis de résoudre quelques problèmes qu'elle pose, comme B. Gèze l'a récemment tenté (1962), sans toujours distinguer ce qui revient à l'hydrologie de la zone noyée, entre le niveau de base karstique et le niveau horizontal des plus basses résurgences, et ce qui revient à la circulation karstique proprement dite, dans les galeries plus larges de la zone amphibie. En particulier, nous savons que les eaux de cette zone basse du système karstique constituent un ensemble ayant certaines propriétés des nappes phréatiques: horizontalité du niveau, diffluences, extension généralisée sous tout un plateau, ce qui fait qu'il peut alimenter plusieurs sources périphériques; cas du Causse de Gramat, des plateaux de la Garrigues de Montpellier, du Dachstein autrichien où la technique de coloration par les spores du lycopode a montré que la 
nappe karstique avait une alimentation générale depuis la surface du plateau et se vidait dans le nombreuses sources vauclusiennes du pourtour.

De plus en plus, cependant, des exploitations atteignent cette zone noyée: c'est le cas des plongées souterraines, et des explorations après pompage (Cavaillé et Marty).

Les observations directes montrent alors des galeries ayant souvent des grandes dimensions, creusées sous l'eau, et tendant, à partir d'une diaclase ou d'un joint originel, à la régularisation en section ovale ou circulaire.

On peut même observer les formes de détail montrant comment agit la corrosion, suivant la nature du calcaire; on y étudie des cupules, des ondulations, des «vagues d'érosion» comme formes mineures; des niches, des cloches, des «marmites de géant» dans toutes les directions, comme formes moyennes; des coupoles, des niches, des banquettes, comme formes majeures. On est surpris de constater que certaines de ces formes, qui étaient attribuées à l'érosion mécanique, sont dans ces conduits toujours noyés, des formes de corrosion.

\section{$d$-Les galeries amphibies du système karstique}

Ces caractères des galeries de la zone noyée ne se retrouvent qu'en dessous du niveau horizontal passant par les plus basses résurgences qui ceinturent les plateaux. Or, il y a tout un ensemble de galeries subhorizontales, immédiatement au-dessus de ce plan qui constitue la «zone spéléologique» par excellence du système karstique. Ce sont les galeries empruntées par l'eau au-dessus de l'étiage, alimentées irrégulièrement, selon les précipitations tombant sur le plateau, et se vidant vers l'aval par les résurgences pérennes et par les sources intermittentes des trop-pleins.

Alternativement vides et pleines d'eau, elles s'étagent sur une épaisseur variable. On a une idée de cette épaisseur par les différences de niveau considérables qui peuvent atteindre 30,50 ou 80 mètres, que les spéléologues ont observées au fond de certains gouffres, selon l'époque de leur exploration; très nombreux sont les cas où, par temps de sècheresse, on a pu poursuivre l'exploration des avens ou des grottes situées à l'amont des systèmes karstiques sur des dizaines de mètres de profondeur supplémentaire: l'Igue de Planagrèze et la grotte de Lombard en Quercy, l'aven de Hures, et la plupart des Grandes cavités du Vercors sont dans ce cas (Grotte de la Luire).

Dans cette zone alternativement sèche et noyée, les conditions de la spéléogénèse sont très favorables à la formation des galeries. Ici, il 
peut y avoir vraiment des galeries en charge, ou en conduite forcée, des directions privilégiées; par exemple une diaclase plus large, une cassure tectonique, un fond de synclinal concentrent les eaux, et la corrosion augmente avec la vitesse et le débit. Toute galerie qui commence à s'élargir devient apte à s'élargir davantage; ainsi se forment les cavités récemment décrites par Renault, en conduite forcée pour les plus basses, en écoulement libre pour les plus hautes. La section des premières tend à devenir circulaire, mais est le plus souvent ovale dans le sens des diaclases ou des joints originals; celle des secondes s'allongent vers le bas, et aux actions de la corrosion s'ajoutent celles de l'érosion (marmites de géants).

Ici se rencontrent encore les différentes types de microformes de parois, celles de dissolution comme les cupules, les facettes, les coups de gouge; mais il est souvent difficile de distinguer ce qui s'est établi sous l'eau, de ce qui se forme vraiment dans le régime amphibie; les formes d'érosion comme les marmites du fond, peutêtre les stries ou les cannelures plus ou moins hélicoïdales, sont certainement des retouches de la galerie primitive mais elles sont très rares.

Le renouvellement de l'eau, venue très vite de la surface et donc encore agressive, augmente la corrosion. La vitesse du courant permet le transport des matériaux de remplissage, provenant des impuretés de la roche encaissante et donc le plus souvent fins, sauf dans le cas des calcaires sableux ou des roches à silex.

Les dimensions des galeries sont d'ailleurs limitées par l'importance de ces impuretés: celles-ci peuvent s'accumuler dans les zones basses, combler les siphons, et obliger l'eau à ouvrir d'autres passages. La présence de cette «argile primordiale» modifie constamment le dessin du réseau, provoque des mises en charge locales, crée des bouchons, interrompt les écoulements; ainsi «les galeries actives» comme les appellent les auteurs, ne sont-elles pas toujours des artères principales du drainage. Il n'y a pas forcément, en arrières des résurgences, un réseau de galeries affluentes donnant sousterre le réseau hydrographique que les rivières aériennes dessinent en surface. Pourtant, par dessus la zone noyée, ce réseau tend à se constituer; la grotte de Miramont en offre deux exemples superposés: l'un actif, l'autre sec.

\section{e-Le système karstique élémentaire}

Sous tous les plateaux calcaires, quelle que soit leur altitude relative, leur composition lithologique, leur histoire morphologique, on retrouve donc ce système karstique. En admettant une certaine stabilité des vallées qui constituent le niveau de base local et qui, dans les conditions 


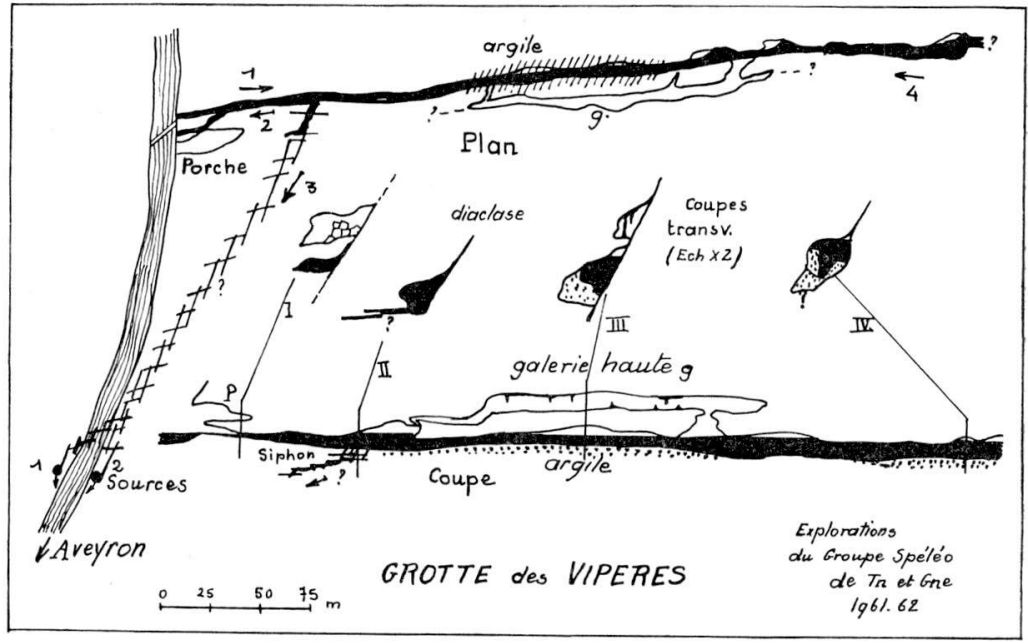

Fig. 3: Grotte des Vipères. Cet exemple nous montre la présence d'une circulation au-dessous des rivières (sources 1 et 2 de part et d'autre de l'Aveyron), et l'existence des grosses galeries aux environs du niveau actuel des rivières. Il montre aussi la localisation des dépôts argileux au contact des eaux boueuses venues de l'Aveyron par l'entrée, et des eaux du Causse venues de l'intérieur. - Sens des courants: 1, en crue de l'Aveyron; 2, en crue locale des eaux souterraines; 3 , vidange de la galerie sous le lit de la rivière; 4, arrivée des eaux infiltrées sous le Causse.

actuelles de l'érosion aérienne, semble réalisée partout, ce système a le temps de s'établir.

Il comporte différents éléments: chenaux d'infiltration en surface; cheminées plus ou moins profondes selon l'altitude relative du plateau; galerie larges, explorables, tendant vers la conduite circulaire et se disposant plus ou moins en réseau organisé; ouvertures très irrégulières, diaclases, poches de corrosion, formant des chenaux anastomonés sous le niveau hydrostatique régional. Toutes ces formes peuvent être appelées «formes primordiales du système karstique»: leur creusement n'a en principe, d'autre limite que la disparation totale de la masse calcaire par dissolution. Celle-ci, sous climat froid, est plus importante dans la partie supérieure du système karstique (lapiaz); sous climat chaud, à sa partie inférieure, aux environs du niveau hydrostatique (formation des poliés). Sous nos climats, elle semble être assez régulièrement répartie sur toute l'épaisseur des plateaux. 


\section{Evolution du système karstique élémentaire}

Dans la réalité, ce système karstique ne fonctionne pas longtemps selon ces principes simples. En effet, interviennent un grand nombre d'influences qui modifient son établissement, et qui règlent son évolution.

Les unes sont d'ordre interne, et résultent de l'évolution même du système karstique, toutes les autres causes restant stables; ce sont le remplissage, le concrétionnement, les éboulements.

D'autres sont d'ordre externe; c'est l'influence de la géologie: présence d'un niveau imperméable sous la masse calcaire, conditions tectoniques. C'est aussi l'influence de la géographie: caractères du réseau hydrographique aérien, importance des formations superficielles.

Enfin, les plus grandes perturbations apportées à l'édification du système karstique sont dues à l'instabilité du niveau des plus basses sources vauclusiennes. Or, celles-ci suivent fréquemment (pas toujours) et avec plus ou moins de retard, l'enfoncement des vallées qui constituent le niveau de base local; le système karstique évolue selon cet enfoncement.

Enfin si, au contraire, le niveau de base s'élève ou si le climat change et devient impropre à la karstification, le système karstique se fossilise. Il pourra s'établir ensuite un nouveau système karstique tout a fait indépendant du précédent.

\section{Evolution propre du système karstique}

Mème dans le cas où les conditions restent très longtemps stables, où le massif calcaire est homogène, la forme des galeries du système karstique évolue, plus ou moins vite selon la nature lithologique de la roche encaissante. Cette évolution tient aux conditions mêmes du travail des eaux souterraines.

a-Rôle de remplissage

Le calcaire dissous laisse un résidu plus ou moins important selon sa pureté. Une grande partie de ce résidu se dépose sur place, souvent même sur les parois des galeries noyées, où il forme une couche homogène. Les plongées dans ces galeries, ou encore les quelques incursions qu'on peut y faire depuis les galeries horizontales, nous montrent la présence de cette couche argileuse qui tapisse toutes les parois. L'argile flocule dans l'eau chargée de calcaire et le remplissage acquiert une certaine stabilité. 
On peut même se demander si cette croûte n'exerce pas un effet protecteur, ce qui augmenterait encore le rôle du courant sur l'intensité de la corrosion. Mais nous n'avons pas de mesures précises pour contrôler cette action.

Les remplissages les plus importants se font dans les cavités de la zone amphibie. Le courant enlève le dépôt argileux d'un point et le dépose en d'autres. Nous avons déjà montré que ce dépôt était surtout conditionné par le mélange des eaux boueuses et des eaux calmes saturées de calcaire (1960).

En plus de l'argile résiduelle, qui peut ne représenter qu'une infime partie du dépôt, les galeries du niveau amphibie recueillent les dépôts apportés de l'extérieur; ces eaux agressives charrient l'argile colloïdale, abandonnent leur charge au contact des réserves riches en calcaire de la zone basse. La présence d'importants amas argileux après les confluents souterrains s'explique par l'arrivée d'eaux boueuses dans une réserve d'eau calme saturée.

Très rapidement, ce remplissage argileux peut rendre étanche le bas de la galerie, d'autant plus vite que, par migration dans la masse, le calcaire des eaux d'imbibition se rassemble et la rend compacte. Il se constitue des dépôts de calcite rameuse à l'intérieur de l'argile, dépôts qui se rassemblent jusqu'à constituer de faux planchers stalagmitiques; le plus haut de ces planchers, au contact de l'air de la galerie, peut devenir très dur.

Ph. Renault a souligné le rôle de ce remplissage dans la corrosion de la galerie; celle-ci s'élargit au-dessus du remplissage, et certaines sections de cavernes s'expliquent par cette évolution.

Les «coups de gouge» ou «vagues d'érosion» sur les parois de cavernes ont pour origine la corrosion ou bien, si l'érosion en est responsable, ils se sont formés bien avant l'évolution de la galerie, avant la fin de la première phase de creusement, c'est à dire en dehors du remplissage important. L'observation d'ailleurs corrobore ce raisonnement; les microformes de paroi sont le plus souvent ennoyées par l'argile, ce qui exclut les érosions.

Plus important est le rôle du remplissage sur la figuration même du réseau. Il peut se constituer de véritables bouchons, et chacun de nous connait des galeries pleines d'eau par suite d'un remplissage argileux. Dans ces conditions la corrosion de la roche est guidée par ces dépôts et, à partir des diaclases originelles, évoluent les méandres, les recoupements de galeries, les diffluences horizontales ou verticales.

Egalement peuvent s'expliquer de cette manière les traces de corrosion au plafond de galeries déjà formées, ce qu'on appelle les lapiaz 
souterrains, et les reprises de concrétionnement, la formation des planchers suspendus, par exemple.

L'étude du remplissage, précisant les conditions de dépôt et leur constitution, est un élément essentiel de la connaissance des grottes: elle n'est pas assez faite, en général, dans la monographie des cavités.

\section{$b$ - Le concrétionnement}

Lorsque la corrosion et l'érosion ont ouvert les galeries, le remplissage provoque une première évolution; certaines parties des galeries peuvent être plus ou moins abandonnées et le concrétionnement intervient.

Notons bien que dès qu'un concrétionnement important se produit, avec coulées, colonnes et formation des planchers, la galerie atteint le stade de vieillesse; elle évoluera, changera de forme et d'aspect sans que s'exercent les processus qui l'ont ouverte.

Les eaux qu'elle peut recueillir, venues de la surface, sont peu importantes; elle se chargent de calcaire en traversant toute la masse et elles peuvent alors édifier un concrétionnement. Parfois même, on a des galeries mixtes, concrétionnées à la partie supérieure, pleines d'eau à la partie inférieure; ces eaux, si elles participent à la circulations générale, sont agressives et empêchent tout concrétionnement; celui-ci s'arrête au niveau de l'eau.

L'arrivée des eaux agressives dans les galeries peut se faire à très grande profondeur. C'est, par exemple, ce qu'a constaté A.Bögli dans le Hölloch.

Le concrétionnement peut se poursuivre jusqu'au colmatage complet de la galerie, selon des modalités en rapport avec la météorologie de la grotte. C'est cette phase de l'évolution que les spéleologues étudient le plus souvent; dans bien des cas, ces fragments de galerie qui ne sont pas forcément plus hauts que les galeries actives, sont les seules connues de ce que nous appelons le système karstique.

\section{$c$ - Les éboulements}

Les processus de remplissage et de concrétionnement sont parfois bouleversés par les éboulements. Ceux-ci se produisent avec une grande ampleur, dans certaines roches bien stratifiées et diaclasées, dans les calcaires en gros bancs où se produit une imbibition par les eaux agressives venues de la surface.

L'éboulement agrandit la galerie localement et peut être à l'origine des dépôts qui interrompent la continuité des galeries. Ils se produisent généralement avant que le concrétionnements ne soit terminé, mais alors que la grotte a atteint sa pleine maturité. 


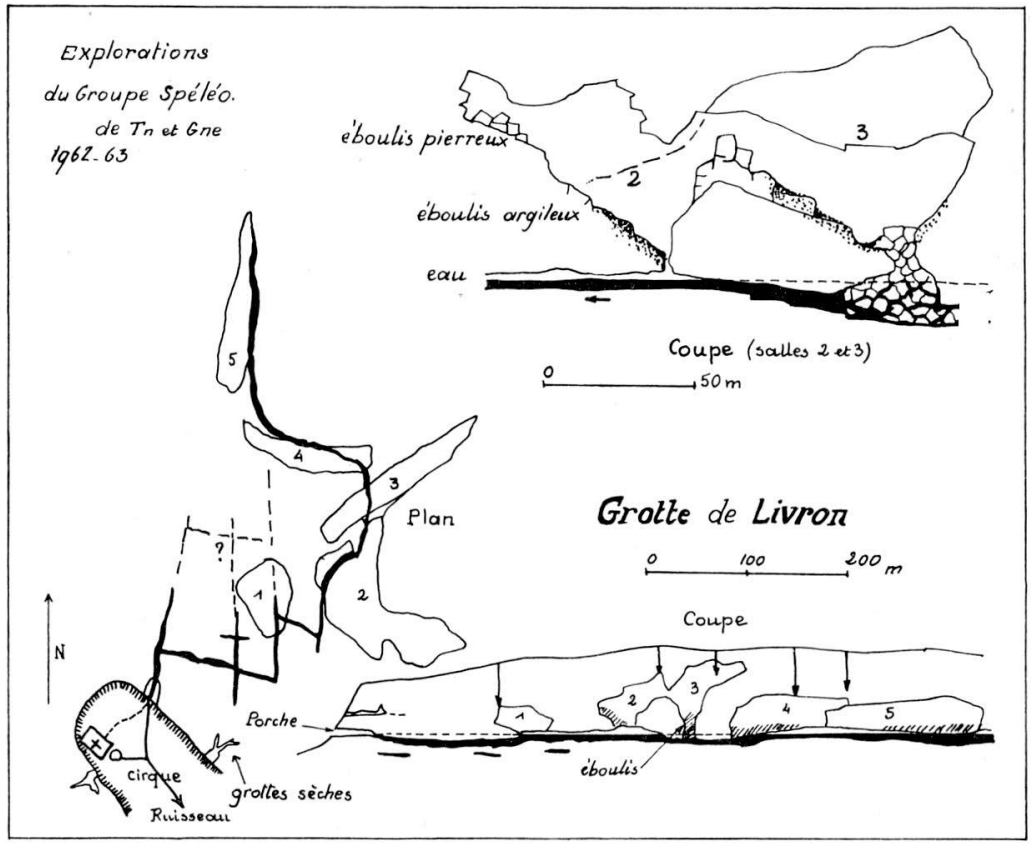

Fig. 4: Grotte de Livron, montrant la formation des salles d'éboulis audessus de la galerie pleine d'eau du système karstique actuel. - 1 à 5, salles d'éboulis. Les flèches à leur plafond indiquent les arrivées d'eau agressive.

Noter le tracé de la galerie actuelle conforme à la lithologie.

Les éboulements et les concrétionnements le long d'une galerie peuvent être contemporains, les premiers correspondant à une arrivée d'eau agressive qui imbibe la masse encaissante, les seconds à une arrivée d'eau saturée, qui a traversé plus lentement la masse calcaire supérieure.

Ces éboulements modifient beaucoup la forme originelle de la galerie primitive. Ils peuvent se faire à la voùte, ou encore sur une paroi. On reconnait souvent, d'un coté de l'éboulement, l'ancien tracé de la galerie, en tunnel ou en diaclase.

On peut se demander si, dans une région donnée, les éboulements qui affectent les cavités se correspondent: par exemple, en Quercy ou sur les Grands Causses, on observe des éboulements de falaise qui se sont produits à la même période, à un niveau identique au dessus des rivières actuelles. Les éboulements dans les galeries pourraient alors correspondre à une période de tremblements de terre qui auraient 
bouleversé la masse calcaire déjà désagrégée par les infiltrations corrosives.

Ou encore, les éboulements souterrains peuvent correspondre à une phase climatique générale, plus froide, qui aurait provoqué l'arrivée en profondeur d'eau agressive: ils auraient une origine analogue à celle qui provoque les éboulements au fond des puits à neige des hauts plateaux du Vercors ou du Dachstein autrichien.

En Quercy, toutes les grandes grottes horizontales du niveau inférieur présentent ainsi de vastes salles d'éboulis à travers lesquels suinte encore de l'eau.

Car l'éboulement ne termine pas la vie de la cavité. Fréquemment, l'éboulement continue, après sa chute, à se désagréger sous l'action des eaux qui l'ont provoqué: salle terminale du Capucin, salle du Livre au Réveillon etc. On voit alors les blocs éboulés se désagréger sur place peu à peu, et la salle se régularise; si l'arrivée d'eau agressive tarit, les derniers restes des éboulements se recouvrent de concrétions et l'ensemble se consolide à nouveau; telles sont les vastes salles de nos grottes touristiques, Cabrerets, l'aven Armand, l'aven d'Orgnac... Il est alors difficile de reconstituer la galerie primitive, dont la dimension n'a pas de commune mesure avec les salles qui s'ouvrent sous nos yeux. La salle du Chaos, à Padirac, donne aussi un bon exemple de ce processus.

Parfois ces éboulements se produisent à la voûte, le long de cheminées verticales qui amènent l'eau de la surface: si les blocs sont corrodés par le bas, l'affaissement se produit d'une manière continue; ainsi peuvent s'ouvrir des gouffres, ou peuvent se former les dolines d'effondrement taillées comme à l'emporte-pièce à la surface du Causse: Padirac en est l'exemple classique. Ce processus a d'ailleurs été étudié par B. Gèze (1953).

Il faut cependant souligner le fait que, dans la formation des salles ou des cheminées d'effondrement, les blocs éboulés doivent être évacués par le bas. Cet enlèvement se fait par corrosion, dissolution sur place, et non pas par transport: ainsi de minuscules galeries peuvent brusquement s'élargir en vastes salles, que la galerie soit verticale comme à l'aven Armand ou horizontale comme à Cabrerets et à Padirac.

\section{d-L'érosion de la surface}

On a signalé à diverses reprises la remarquable stabilité de la surface des plateaux calcaires. Cependant, cette surface baisse par corrosion des eaux de pluies. Les mesures faites après les pluies, dans les flaques qui restent à la surface, montrent qu'assez rapidement, avant de 
s'infiltrer, cette eau contient des quantités appréciables de calcaire qui indiquent une corrosion superficielle mesurable (A. Cavaillé, 1953).

Cet abaissement de la surface peut mettre à jour les bouches des cheminées descendantes du système karstique. Les gouffres en diaclase de petites dimensions, que l'on rencontre sur les plateaux du Quercy, ou sur les Grands Causses, n'ont pas d'autre origine; leur position est quelconque par rapport à la topographie de la surface. Cependant, beaucoup d'entre eux, ayant subi l'influence des climats périglaciaires, ont évolué et leur bouche s'est plus ou moins modifiée. D'autres sont cachés sous la terre arable, ou encore ont été couverts par les hommes; la densité de tels gouffres est beaucoup plus grande qu'on ne le croit, même si leurs faibles dimensions les rendent impénétrables.

En tous cas, la corrosion de la surface des plateaux n'empêche pas le système karstique d'évoluer normalement. Comme nous l'avons déjà signalé, cette évolution conduit à l'affaiblissement de toute la zone calcaire aux environs du niveau hydrostatique local. Cet affaiblissement doit se marquer, aux termes de l'évolution par des effondrements considérables qui se traduisent dans la topographie extérieure par des dolines, des reculées du côté des résurgences, et des poliès du côté de l'alimentation du système karstique. Mais ces formes extérieures ne se manifestent qu'après une longue évolution; dans le karst de nos pays elle est loin d'être menée à son terme, par suite de la lenteur des phénomènes de corrosion, et par l'instabilité des niveaux de base qui ont plusieurs fois varié depuis le quaternaire.

\section{B - Influence de la géologie}

L'établissement et l'évolution du système karstique tel que nous venons de les décrire ne se font que lorsque la masse calcaire est homogène et est plus basse que le niveau de base local.

\section{$a$ - Le plancher marneux}

Ces conditions sont assez fréquemment réalisées. Mais souvent le plateau calcaire repose sur un plancher de marnes imperméables. Ce plancher constitue le niveau de base karstique; il peut être au-dessus du niveau hydrostatique local. S'il est au-dessus, les galeries amphibies du système s'établissement au niveau des marnes, mais très souvent la limite n'est pas nette.

En effet, dans la plupart des cas, les marnes sous-jacentes peuvent être le siège d'une circulation karstique: il s'établit à la longue des galeries dans la marne elle-même. Il en est ainsi pour le toarcien du Quercy, où les niveaux marneux de la partie supérieure ne contiennent 
que 40 à $60 \%$ de calcaire. Dans ces conditions, les galeries ne s'agrandissent que par des effondrements. Rares sont les cas où, au cours des explorations, on peut reconnaitre la paroi de corrosion de la galerie; par contre, les éboulements sont nombreux, les blocs se délitent en donnant beaucoup d'argile que l'eau étale sur le plancher des salles avant de les emporter. Localement, aux ruptures de pente, une érosion peut s'exercer avec chutes reculant par érosion régressive. On obtient alors des galeries très irrégulières en plan et coupe donnant des lignes brisées à angles droits, et qui ont été bien étudiées par Ph. Renault (1962).

Ce n'est que lorsque la galerie est abandonnée par les eaux qu'elle prend une forme figée, par les concrétions qui moulent les parois et soudent les éboulis; nous avons alors des galeries au-dessous de la limite habituelle; il en est ainsi dans beaucoup de pertes des Causses du Quercy. La condition essentielle pour que de telles galeries s'ouvrent dans les marnes, qui ne présentent pourtant en surface aucune forme karstique, c'est que le niveau général des eaux soit assez bas pour que s'évacuent les grosses masses d'argile résiduelle provenant des éboulements des galeries.

Tous les explorateurs des Causses du Quercy, de Padirac à la rivière du Bosc, doivent vaincre les énormes difficultés que créent ces dépôts argileux, venus des argiles sous-jacentes et aussi souvent apportées de l'extérieur par les pertes.

\section{$b$ - La tectonique}

On a souvent insisté sur le rôle de la tectonique qui détermine l'emplacement des réseaux, au fond des synclinaux, la forme et la dimension des galeries, dans la mesure où la tectonique est responsable des diaclases. Les conditions tectoniques peuvent donc organiser les réseaux du système karstique, déterminer les lignes directrices, en particulier l'emplacement des résurgences et des galeries principales qui y aboutissent.

Mais il faut insister sur le fait que cette dépendance du karst et de la tectonique est loin d'être totale. P. Dubois a étudié les karsts barrés du Languedoc, qui semblent bien être soutirés par delà le mur de la faille-limite. B. Gèze (1962) cite le cas de la source du Lez, de la source des Cent-Fons (Hérault), de la rivière de Théminettes, où les expériences de coloration ont montré que les réseaux du système karstique traversaient la lèvre surélevée de la faille et le bloc marneux qu'elle dénivèle.

Un autre exemple peut être donné par les pertes du synclinal de Mémer, dans le Causse de Limogne. Ces eaux, rassemblées par la gout- 


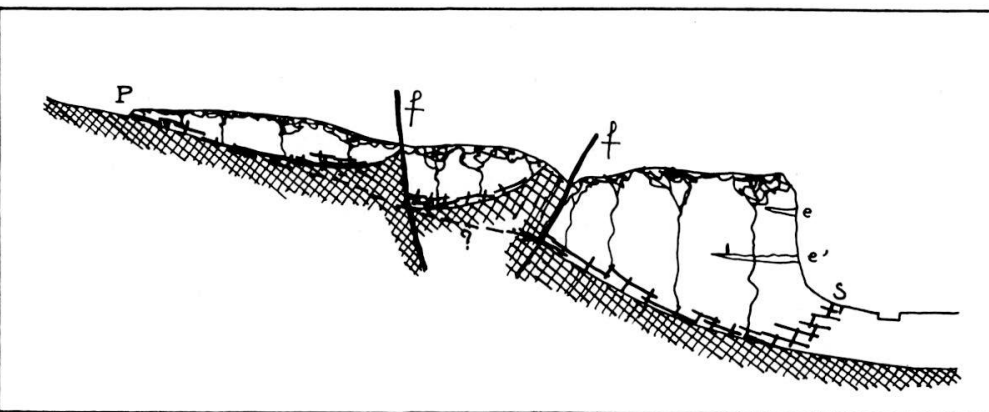

Fig. 5: Influence de la tectonique. Coupe SN du synclinal de Memer (Lot) vers la source de Lantouy. - P, pertes de Puylagarde; f, failles de Laramière; $\mathrm{S}$, source de Lantouy; e, e', anciens systèmes karstiques.

tière du synclinal vont alimenter la source de Lantouy à $25-30 \mathrm{~km}$ vers le nord. Or, cette gouttière synclinale sud-nord est interrompue à Laramière par plusieurs failles est-ouest qui font affleurer le toarcien au milieu du Causse. Les galeries de Lantouy traversent donc le compartiment marneux, mais difficilement; tous les hivers, les galeries d'amont sont noyées par les eaux qui traversent peu à peu les pertuis marneux inconnus (fig. 5).

\section{C - Influence de la géographie}

Les phénomènes externes, ceux qui ont modifié la surface de la terre, et que la géographie étudie, ont bien entendu influencé l'établissement et l'évolution du système karstique; à l'inverse, le modelé karstique constitue un paysage bien particulier, dû justement a l'enfouissement des eaux en profondeur.

$a$-Les vallées sèches

Dans les conditions différentes des climats passés, la surface des plateaux a évolué autrement que sous le régime karstique qu'ils connaissent; ainsi ont pu se former ces curieux réseaux de vallées sèches, qui sont si caractéristiques en Quercy, mais que l'on retrouve dans tous les plateaux karstiques de nos pays, pendant des phases climatiques périglaciaires (R. Ciry).

Les dolines de dissolution, les plus nombreuses, les groupements de dolines, les grands bassins karstiques plus ou moins influencés par la tectonique et que l'on retrouve, comme des reliques d'une évolution ancienne, sur les Grands Causses ou les Plans de Provence, contribuent à modeler le relief des plateaux karstiques. 
Ces vallées et ces bassins peuvent donc opérer, dès la surface, une première concentration du draînage; les cheminées verticales descendant sous les bassins, même hauts, deviennent plus importants que leurs voisines; les effondrements, dont elles sont le siège, peuvent y ouvrir plus facilement des gouffres de par le bas: on s'explique alors que la densité des avens, des igues ou des scialets soit plus importante près des vallées; cela ne veut pas dire, comme on le voit écrit très souvent, que ces gouffres sont "les anciens puits absorbants» de la vallée active. Très rares sont ces gouffres absorbants creusés par un ruisseau actif: il faut toujours en trouver la vallée sèche en amont, avec un bassin de réception aérien important, que justement la karstification ne permet pas. La formation des pertes suppose des conditions particulières: elles ne se forment plus, elles ne se sont jamais produites dans le passé, directement, sur les plateaux karstiques. Les exceptions à cette règle (comme Roc de Corn) sont toujours facilement explicables par la présence locale d'un revêtement argileux sur le Causse.

Mais l'action des vallées sèches sur le karst profond peut être plus grand encore, surtout lorsqu'il s'agit de vallées sèches profondes, pas très éloignées du niveau hydrostatique actuel. En dessous d'elles, lorsqu'elles étaient actives, s'est organisé un réseau plus important de diaclases et de conduits, comme il s'en organise un encore sous les rivières allogènes qui traversent, dans les gorges plus ou moins profondes, les plateaux karstiques. La vallée devenue sèche, ces conduits sont plus ou moins désaffectés, mais ils peuvent être sporadiquement actifs; c'est ainsi que sous la plupart des vallées un réseau s'individualise dans le système karstique général; chaque vallée sèche a des écoulements sous-jacents, qui se vident par des résurgences particulières le long des grandes vallées; cette règle se vérifie chaque fois en Quercy, le long des quatre vallées transversales qui découpent le Causse. Elle se vérifie aussi le long des gorges des Grands Causses (J. Rouire, 1963).

\section{$b$ - Les pertes}

Dans des conditions particulières de la géologie, les ruisseaux établis sur les terrains non karstiques se perdent lorsqu'ils arrivent à la limite du Causse. Aucune autre région en France n'est aussi riche en pertes que la bordure sud-ouest du Massif Central, où on peut en compter des centaines. Généralement, en effet, le relief de côtes est développé à la limite amont des plateaux calcaires, et les cours d'eau subséquents ont soutiré les pertes. Mais on connait des pertes, dans d'autres conditions morphologiques, dans la plupart des régions karstiques. 
Parfois, ces pertes se produisent après que le ruisseau a dépassé la limite du calcaire, prolongeant plus ou moins longtemps, après l'assèchement général, la circulation dans la partie amont des vallées sèches. Ce fait s'explique par le colmatage alluvial du fond de la vallée; ce colmatage disparaissant peu à peu, usé par l'érosion, la perte recule vers l'amont jusqu'à la rencontre de la roche perméable. Ainsi peuvent s'établir, à l'amont, des vallées sèches, des "vallées aveugles» qui sont, dans le relief, les symétriques des «reculées» établies vers l'aval.

Quoiqu'il en soit, ces pertes introduisent dans le système karstique des directions particulières, et une quantité considérable d'eaux agressives, surtout lorsque le ruisseau aérien coule sur un sol acide et forestier. Il s'établit ainsi après la perte, un réseau particulier que l'influence de la vallée sèche, s'exerçant comme nous venons de le décrire, peut guider. Mais la dépendance de ce réseau particulier vis-à-vis des anciennes circulations aériennes, n'est pas obligatoire, et B. Gèze cite de nombreux exemples de l'indépendance des deux circulations; les captures souterraines d'une vallée vers l'autre, parfois d'un bassin vers un autre, sont des cas assez fréquemment décrits...

Le rôle des pertes dans l'évolution du système karstique est important, également, par l'abondant remplissage que ces pertes peuvent apporter dans les galeries noyées de la nappe en réseau.

Ce remplissage peut être constitué par des cailloux roulés, des graviers, des sables, le plus souvent formés de roches non calcaires. C'est ainsi que l'aven Viala, ouvert dans une série redressée où se succèdent des zones calcaires étroites emprisonnées dans de larges zones schisteuses à filons de quartz, montre un important remplissage de galet de quartz et de schistes, souvent peu émoussées. Au bas des diaclases et des puits comblés par ces graviers, la corrosion ultérieure a dégagé une galerie à la voûte de laquelle ces dépôts semblent comme suspendus; ils montrent d'intéressantes intercalations de varves, et une succession de planchers stalagmitiques. Ils ont manifestement influencé l'évolution des formes de la galerie actuelle.

Finalement, l'arrivée des pertes dans un système karstique agit sur l'évolution des galeries préexistantes de deux manières, qui tiennent à l'irrégularité des débits et de la composition des eaux; par temps de crues, elles provoquent des corrosions importantes et des débuts d'érosion; par leur charge solide, elles peuvent constituer des bouchons imperméables et modifier beaucoup le dessin du réseau primitif. Les importants réseaux du Mas de Bénac et de Foissac, en Rouergue, sont de bons exemples de cette intéraction des eaux venues du plateau et des apports des pertes, constituant un inextricable ensemble de 
galeries d'évolution très diverses et cependant établies sur un même niveau.

\section{$c$ - Les formations superficielles}

$\mathrm{Au}$ cours de leur histoire morphologique, nos plateaux karstiques ont été recouverts de formations superficielles imperméables: sidérolithique, argiles à graviers, argiles à silex, loess, etc. Plus ou moins déblayées par l'érosion ultérieure, selon leur àge et leur position topographique, ces formations superficielles constituent le plus souvent des lambeaux entre les vallées sèches; ce fait accentue la concentration du drainage aérien sous les vallées et sous les dolines. Il peut même exister des pertes locales en plein Causse, une concentration du draînage aérien se produisant à la faveur d'un de ces affleurements imperméables. C'est une des causes qui augmentent l'interdépendance du karst et de l'érosion aérienne.

Les dépôts superficiels ont un effet indirect sur la forme des galeries et leur développement, effet qui se trouve aussi à la suite des pertes de rivière. Dans ces conditions, il pénètre dans la masse calcaire des quantités considérables de transports solides. L'analyse granulométrique, l'examen binoculaire des sables permet d'ailleurs de trier ce qui revient, dans le remplissage, au transport des ruisseaux enfouis et aux résidus locaux de la dissolution de la roche.

Arrivés au contact des réserves d'eau saturée de calcaire dans les zones profondes du système karstique, ces dépôts floculent et les conduits peuvent s'obstruer totalement. Ce fait concourt à isoler encore mieux le réseau de galeries à l'aval des pertes, et, en principe, le temps final de l'évolution d'un réseau karstique après une perte serait l'ouverture d'un tunnel à travers toute la masse calcaire; cela se produit dans des conditions particulières, mais dans la plupart des cas le réseau de la perte se disperse, se désagrège dans les conduits inférieurs du système karstique général.

On voit donc que le système karstique qui existe sous les plateaux à tendance à s'organiser, à se fragmenter, en fonction des conditions extérieures: se créent ainsi les réseaux karstiques des pertes et des résurgences que le spéléologue peut explorer et étudier selon son dégré d'évolution. Mais ces «réseaux» mettent longtemps à s'individualiser, ou bien ils se forment dans les zones que les gouffres ne permettent pas d'atteindre. Dans ces deux cas, le spéléologue ne peut pas observer directement, mais il peut raisonner sur des éléments importants: coloration, débit, température et trouble des eaux, conditions géologiques particulières. Il reconstitue indirectement ainsi les caractères hydrologiques d'une région calcaire. 
Les expériences de coloration montrent souvent, en effet, la complexité des réseaux souterrains, selon la période où elles sont effectuées. Ainsi, des colorations faites par temps de sécheresse peuvent montrer des relations directes entre une perte et une résurgence; la mesure des débits prouve d'ailleurs, le plus fréquemment, que la résurgence restitue les eaux de la perte augmentées d'un certain volume d'eau mis en réserve dans la masse calcaire.

Si la coloration est faite par temps de crues à la perte, les indications qu'elle donne sont différentes; par exemple, les eaux peuvent se disperser pour ressortir dans plusieurs résurgences sur la ceinture du plateau. Il est très rare, donc, que des communications directes s'établissemt, comme si, dans le réseau de fentes l'eau hésitait entre plusieurs directions. Il y a même des cas, où, selon la pluviosité, le sens du courant peut s'inverser dans la même galerie (exemple de la Grotte des Vipères). Les arrivées rapides et irrégulières des eaux déjà concentrées des pertes augmentent beaucoup cette indétermination des écoulements souterrains.

\section{D - Instabilité du niveau de base local}

Nous avons supposé jusqu'ici, dans l'établissement et l'évolution du système karstique, la stabilité des niveaux de base, qui se marque par exemple par la constance de niveau de la plus basse source du système. L'évolution du système karstique dépend de la durée de cette stabilité: les grandes galeries de la zone amphibie, les tunnels, les gouffres d'effondrement du plateau, la formation des reculées et des vallées aveugles, la présence des dolines profondes, le creusement des poliès sont les phénomènes successifs qui, sous un même climat, et pour une même roche, marquent les étapes du karst en fonction de la durée de cette stabilité.

Les classifications de Cvijic, holokarst, mérokarst, etc... dépendent beaucoup plus de l'histoire morphologique que des conditions actuelles du climat et de la géologie.

C'est ainsi que la plupart des karsts de nos plateaux sont incomplets, de faibles dimensions, parce que les niveaux de base ont varié au cours du quaternaire, comme le montrent les terrasses étagées le long des vallées fluviales.

\section{a-L'abaissement des résurgences}

Ce sont les sources, point de soutirage des systèmes karstiques qui constituent le niveau piézométrique aux environs duquel, en dessous ou en dessus, s'organisent les réseaux de galeries. 
Or, le niveau des rivières peut baisser sans que la source elle-même soit modifiée, tant que le lit du cours aérien ne recoupe pas les chenaux inférieurs du système karstique. Par exemple Fontaine l'Evèque est restée suspendue au-dessus du Verdon, la Clamouse au dessus de l'Hérault, les sources des gorges au-dessus de l'Ardèche; il y a bien entendu des conditions locales pour expliquer que les sources restent ainsi suspendues au-dessus des vallées, mais aussi ce fait général, que les rivières méditerranéennes ont très récemment et d'une façon assez brusque, approfondi leur cours.

Au contraire, les rivières du Sud-Ouest aquitain se sont approfondies plus progressivement, et depuis plus longtemps; les résurgences actuelles se sont établies au niveau même des vallées humides; ce sont celles-ci qui constituent en fait le niveau piézométrique du système karstique.

Tant que les chenaux inférieurs ne sont pas recoupés par le lit de la riviére, la résurgence perchée continue à commander l'évolution du système karstique qui l'alimente. Cela arrive aussi lorsque son fond est colmaté par le remplissage argileux, ou que son débouché est maintenu par un dépôt de tuf. Le réseau amont peut alors évoluer sur place pendant plus longtemps et mieux s'organiser: c'est dans de telles conditions que le spéléologue peut s'attendre à l'exploration de réseaux importants.

Plus fréquemment, la source suit l'enfoncement de la rivière et la zone alternativement sèche et humide baisse avec plus ou moins de retard. Un réseau de galeries nouvelles a tendance à se former en fonction de la nouvelle sortie, au-dessus de la zone profonde à chenaux constamment mouillés qui se reforme ou s'approfondit aussi rapidement que baisse la rivière. Ces galeries jeunes se forment d'abord dans les zones où l'écoulement souterrain est le plus concentré; il se constitue ainsi, d'abord, la bouche remontante de la nouvelle résurgence, puis un nouvel étage partant de l'aval vers l'amont du système karstique. Les deux étages peuvent communiquer par des galeries de raccordement subverticales.

Il y a beaucoup de chances pour que la nouvelle source, ouverte en fonction du niveau de base actuel s'ouvre, à un niveau plus bas, aux environs de l'ancienne; c'est, en effet, dans cette zone que la masse calcaire est le plus «imbibée», que les diaclases et joints profonds sont le plus agrandis par l'action des eaux de la zone basse. Il se constitue alors des niveaux de grottes étagées dans le même cirque, ou dans le même secteur de la vallée (souvent un méandre concave, comme le long de l'Ardèche). 


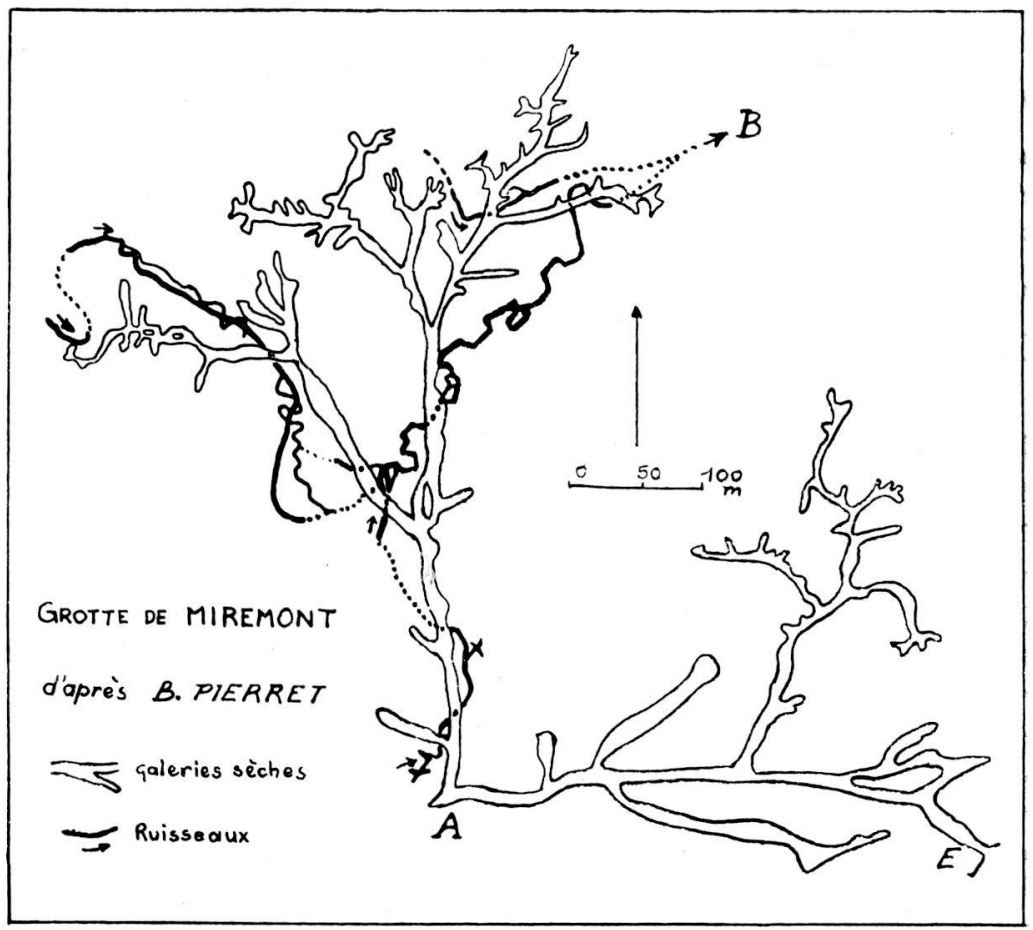

Fig. 6: Grotte de Miramont, montrant la superposition des galeries de deux systèmes karstiques successifs. - E, entrée actuelle; A, vidange possible du système karstique ancien; B, vidange du système karstique actuel.

Mais il peut arriver que la nouvelle source se forme très loin de la première, en un point plus bas du réseau aérien. Qui connaitra jamais les anciens écoulements de la fontaine des Chartreux? Peut-être les ruisseaux souterrains des Igues d'Arcambal à 5 ou $6 \mathrm{~km}$ à l'amont, dans la vallée sèche de Concots. A mesure que le réseau aérien se grave dans la masse calcaire, les grandes sources, celles qui seraient capables de soutirer tout le système karstique si la stabilité se prolongeait longtemps, ont tendance à migrer vers l'aval des bassins hydrographiques.

Mais les sources peuvent changer de place au cours de l'enfoncement général des rivières; celles-ci peuvent recouper les chenaux noyés du système karstique en des points éloignés du soutirage primitif, par exemple sur le versant opposé du plateau, et il s'opère alors un renversement du drainage souterrain.

Le magnifique plan de la grotte de Miramont dressé par B.Pierret (1949), montre nettement ce renversement du draînage souterrain dans 
deux étages différents de galeries horizontales. Le débouché du plus vieux système karstique n'est pas découvert, et il semble que l'auteur n'ait pas déterminé son emplacement réel.

$b$ - Abaissement progressif ou saccadé

Le niveau de base des rivières, que suit avec un temps de retard le niveau piézométrique du système karstique, peut s'abaisser progressivement ou par saccades. Dans le premier cas, le niveau de galeries du système karstique ne se forme pas: les réseaux n'ont pas le temps de s'organiser. Dans le cas d'enfoncement saccadé, des réseaux s'organisent à diverses altitudes, et entre eux une relation altitudinale peut-être établie. En fait, c'est le plus souvent un régime mixte qui s'installe. $\mathrm{Au}$ cours du quaternaire, dans la plupart de nos plateaux karstiques, l'enfoncement a été trop rapide pour que s'établissent de véritables niveaux de galeries sèches: il y en a peu dans les Grands Causses; quelques étagements s'amorcent en Quercy, sur trois niveaux, comme je l'ai montré; les plateaux languedociens et cévenols montrent des étagements plus nets; on en a signalé en Bourgogne (H.Tintant) et dans les Ardennes (C. Ek).

\section{$c$-Les galeries sèches}

Les spéléologues explorent le plus souvent ces galeries sèches des anciens systèmes karstiques dans les grottes horizontales ou les gouffres verticaux abandonnés. Leur évolution est bien connue.

Notons, tout d'abord, que fréquemment les grottes de versant sont les anciennes sorties des résurgences; elles sont alors en pente vers l'intérieur du plateau et une circulation très locale peut les avoir affectées en sens inverse. Très nombreuses sont les grottes que l'on prend pour des pertes et qui sont en réalité le débouché d'anciens systèmes karstiques qu'un ruissellement inverse sur le plancher a modifiés. La grotte bien connue de Lascaux en est un exemple.

Ces renversements de draînage peuvent aussi affecter des galeries ou des tronçons de galerie de l'ancien réseau; il y a le plus souvent, au plafond ou sur les parois, des traces de l'écoulement qui a ouvert la galerie primitive.

C'est dans ces galeries qu'on observe les diverses étapes du concrétionnement, particulièrement dans les plus basses, où les eaux cheminant à travers la masse du Causse peuvent arriver saturées de carbonate: la plupart des grottes concrétionnées sous nos plateaux, appartiennent au réseau de galeries du précédent système karstique; toutes les formes du concrétionnement peuvent s'y observer selon la nature de la roche encaissante (par exemple, la porosité), selon l'im- 
portance et la nature du remplissage argileux, qui se tasse et est plus ou moins imperméable, selon le climat de la surface et de sa végétation, etc...

Les galeries de ces anciens systèmes sont abandonnées par les eaux qui ont trouvé des cheminées de descente plus rapides pour gagner le niveau karstique nouveau. Les effondrements s'y font plus rares, les anciens sont peu à peu consolidés; même s'ils se produisent, aucun soutirage ne se fait plus par le bas et l'affaissement se stabilise rapidement, dans un chaos de blocs et de colonnes emmélés.

Cependant, localement, des arrivées d'eau anciennes peuvent ètre utilisées temporairement, par dessus les coulées de calcite et les rideaux de concrétions en cascades. Par fortes pluies, l'infiltration retrouve parfois ses anciens chemins et la grotte peut redevenir active. Cela se produit surtout lorsqu'une phase climatique humide succède à une phase sèche, comme cela semble s'ètre produit peu avant l'ère historique dans nos régions: une "gorge de surcreusement» comme dit $\mathrm{Ph}$. Renault, peut alors s'ouvrir dans le plancher stalagmitique et dans le remplissage consolidé de la galerie, par l'effet conjugué de la corrosion et de l'érosion travaillant facilement dans ce matériel tendre: il se forme alors de curieuses formes spéléologiques.

Les grottes de l'étage inférieur du Quercy sont souvent surcreusées; mais actuellement, le surcreusement est arrêté, des gours se forment à nouveau au fond du surcreusement; c'est un indice de plus de l'assèchement récent du climat (depuis le Haut Moyen-Age, semble-t-il).

Les grottes des étages supérieurs ont subi plusieurs fois ces phases alternativement sèches et humides. L'observation des concrétions permet de les préciser, comme l'a fait Séronie-Vivien dans le Médoc. Le concrétionnement n'est pas un phénomène irréversible; la corrosion, par des venues inopinées d'eau agressive le détruit, en mème temps que les remplissages argileux sont érodés; mais cela ne se produit pas fréquemment, ni sur de longs parcours. Plus une grotte est haute en altitude, plus elle a de chances (malchances pour les spéléologues) d'être fragmentée par des bouchons de concrétionnement.

Mais, pour les grottes supérieures assez sèches, et dans certaines conditions microclimatiques, un autre phénomène se produit: c'est la «digestion» des concrétions par l'extérieur avec formation de «mondmilch», et vraisemblablement sous des actions microbiologiques (V. Caumartin). Tout le monde connait ces concrétions rongées des grottes sèches, près de la surface topographique, du Midi méditerranéen aux bordures atlantiques; parfois cette «digestion» est ancienne, d'autres fois elle est actuelle, et on voit alors le revêtement de mondmilch. Parfois elle est dépassée et des concrétions toutes neuves se 
superposent aux ruines des anciennes (Cabrerets) donnant des formes bizarres, difficilement explicables (disques). Cette disparition des concrétions peut alors rouvrir les galeries, nettoyer les anciennes parois du revêtement de calcite; elles apparaissent alors striées de cannelures, vermiculées par les attaques du mond-milch qui produisent des microformes se superposant aux formes initiales du creusement.

Les remplissages argileux ne sont pas attaqués, ni les planchers stalagmitiques trop terreux; la grotte sèche donc est bouchée bientôt. Assez curieusement, ces grottes ne sont pas des formes jeunes; elles on été des grottes concrétionnées que le perobactérium speloei a ruinées; cette action peut être plus ou moins grande selon les climats mais elle parait ètre générale. Nous l'avons observée à 1000 mètres d'altitude en Autriche (Mixnitz); elle existe en Yougoslavie; nos grottes françaises en présentent des cas innombrables, et une photo des grottes de Cuba dans l'ouvrage de A. N. Jimenez en montre sans le dire un bon exemple. Il y a beaucoup de recherches à faire dans ce domaine, quand la grotte devient sénile.

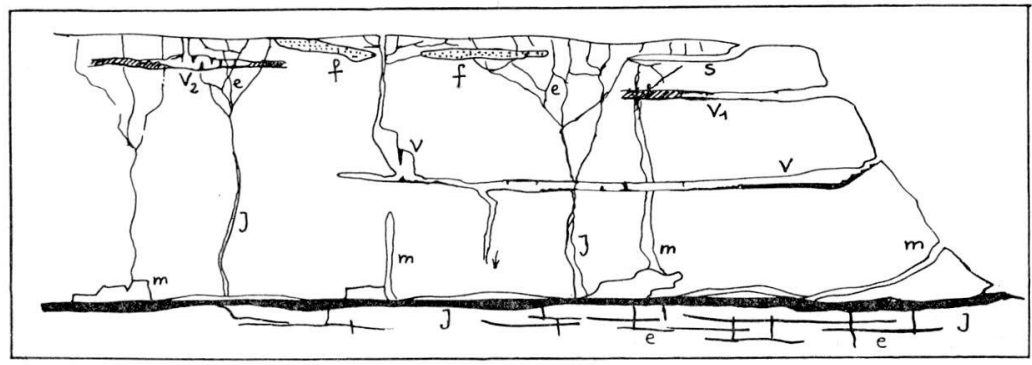

Fig. 7. Karst profond d'une masse calcaire homogène. - e, galeries embryonnaires; J, galerie jeune; m, galeries mûres; V, galeries vieilles; $\mathrm{S}$, galerie sénile; $\mathrm{f}$, galerie fossile.

\section{E-Les systèmes karstiques fossiles}

La succession des systèmes karstiques qui s'observe depuis le quaternaire est responsable de la plupart des grottes de nos pays. Mais les mêmes conditions ont pu se produire dans les ères géologiques passées (Eocène du Quercy, Mio-pliocène du Midi languedocien, etc...). Ces grottes portent, dans leur remplissage, des traces de leur ancienneté; elles sont le plus souvent tout à fait colmatées; ce sont alors de véritables réseaux fossiles, les seuls auxquels il faudrait réserver ce nom. 


\section{Conclusion}

Cette dernière remarque nous amène donc à une question de vocabulaire, qu'il est utile de préciser et qui constituera en fait la conclusion des idées essentielles que nous venons d'exposer (fig. 7).

Nous pouvons établir une classification des phénomènes karstiques, galeries horizontales et gouffres verticaux, en utilisant la notion d'âge de la cavité. Ces dénominations sont sans rapport avec la terminologie davisienne dont on a souvent montré les contradictions. Mais une cavité naît, se développe, évolue, et finit par disparaître, puisqu'elle se colmate le plus souvent totalement. Ces diverses phases de la vie de la grotte sont en rapport étroit avec le développement des systèmes karstiques et leur succession dans le temps au fur et à mesure de l'approfondissement des vallées (les cas inverses d'exhaussement des vallées se produisent aussi, mais ils onts beaucoup plus localisés).

La galerie mûre est celle dont le creusement est terminé, où le remplissage se produit, où commencent les éboulements et le concrétionnement. Ce sont les galeries ouvertes en arrière des résurgences actuelles, dont parfois elles constituent les trop-pleins, les galeries en avant des pertes, dont elles évacuent parfois seulement les eaux de crue, les cheminées verticales plus ou moins calibrées qui y aboutissent, sans toujours s'ouvrir à la surface sous forme de gouffre. En principe, les phénomènes de dépôt et de corrosion s'équilibrent, mais les premiers dominent à la fin de la période, et les derniers sont plus importants au début de la période. Pendant cette phase, les réseaux s'individualisent au sein du système karstique stable.

Ces galeries mûres sont les plus longues, les plus nombreuses, les plus fréquemment explorées par les spéléologues qui y parcourent des kilomètres lorsque les réseaux sont bien individualisés, au-dessus du système karstique régional actuel.

La galerie jeune est celle où les phénomènes de corrosion (et quelques phénomènes de remplissage résiduel) s'exercent seuls; elles sont souvent pleines d'eau, montrent à nu les microformes des parois, et un plan calqué sur la lithologie. Leur section débutant par une fente a tendance à se régulariser et à devenir circulaire. La "galerie en conduite forcée" n'est qu'un cas particulier et terminal de ce stade d'évolution.

Ces galeries sont moins connues, car elles sont toujours siphonantes; les pompages peuvent nous les révéler, et le pittoresque de leurs cupules, de leurs arêtes, de leurs dentelles corrodées ne le cède en rien aux concrétions des galeries supérieures. Telles nous sont apparues la Résurgence de Thouriès ou la Grotte des Vipères. 
Les conduits de la nappe en réseau de la zone inférieure sont les galeries embryonnaires qui pourront venir au jour lorsque l'ensemble du système karstique s'approfondira. Embryonnaires aussi seront les diaclases de la surface, parfois un peu agrandies par la corrosion superficielle des lapiaz.

Au-dessus de la zone amphibie, les galeries des anciens systèmes karstiques constituent un ensemble de galeries vieilles, où le concrétionnement l'emporte sur tous les autres phénomènes, remplissage ou éboulements. C'est dans ce type de grottes que nous trouvons les plus belles de nos cavités, avec leurs coulées toutes neuves, leur écoulement léger d'eau saturée de carbonate qui édifie des gours. Peu à peu, les formes primordiales disparaissent, on ne voit même plus la paroi de la roche encaissante.

Le travail de concrétionnement se poursuivant, la galerie peut être tout à fait colmatée par les dépôts. Le spéléologue s'y fraye difficilement un passage en brisant les rideaux de colonnes et de stalactites; mais le travail de désobstruction est toujours décevant tant que l'on ne débouche pas sur une arrivée d'eau corrosive qui maintient ouvert un fragment de galerie. Souvent, le fond des gouffres aboutit à ces galeries suspendues au-dessus du niveau hydrostatique, que la calcite colmate de plus en plus. Ce sont les galeries mortes qui ne sont accessibles par personne.

Un terme particulier de l'évolution est constitué par des galeries séniles qui s'ouvrent près de la surface. La "pourriture de la pierre» a fait disparaître toutes les concrétions; les parois réapparaissent, couvertes de poussière blanchâtre, qui ternit tout au-dessus du plancher d'argile solidifié.

Séniles aussi sont les diaclases à peine élargies que l'on voit en haut des falaises, ou au creux d'une vallée sèche, seulement modifiées par les phénomènes subaériens, notamment ceux qui sont liés aux dernières périodes froides et humides du quaternaire.

Enfin, un remplissage brusque, lors d'une remontée du niveau de base ou lors de la cessation de toute dissolution du calcaire, peut fossiliser plus ou moins le karst (Llopis-Llado), que les périodes ultérieures de karstification s'exerçant sur la même région reprendront plus ou moins. Ce sont alors des casités fossiles que des accidents locaux ou des travaux humains (carrières des Phosphorites du Quercy) rendront accessibles.

On voit que la terminologie que nous proposons est plus précise que celle qui est habituellement utilisée. Les expressions «galerie active», «réseau actif» recouvrent en fait des formes bien différentes, de la diaclase embryonnaire que la corrosion élargit à la galerie morte 
que le concrétionnement actif colmate. Quant aux termes «réseau fossile», "galerie fossile», que l'on oppose aux précédents, ils sont très fréquemment employés d'une manière impropre, quand ils désignent les galeries mûres ou vieilles des anciens systèmes karstiques. La grotte vit toujours, elle n'est pas fossile, ni mème morte, tant que le spéléologue peut aller y observer les phénomènes.

Notons, en outre, qu'une cavité peut être complexe, d'âge divers, lorsque par exemple elle se développe sur plusieurs systèmes karstiques par une diaclase profonde et plus ou moins élargie (Padirac).

Nous sommes certes loin d'avoir épuisé le sujet; mais la classification des grottes, si elle a tenté beaucoup d'auteurs, ne peut avoir de valeur que si elle est génétique,comme toutes les classifications des naturalistes.

\section{EMPLACEMENT DES CAVITÉS CITÉES}

Igues d'Arcambal

Aven Armand

Perte du Mas de Bénac

Rivière du Bosc

Grotte de Cabrerets

Grotte du Capucin

Source des Cent Fonts

Fontaine des Chartreux

Grotte de la Clamouse

Fontaine l'Evêque

Réseau de Foissac

Source de Lantony

Grotte de Lascaux

Source de Lez

Grotte de Lombard

Grotte de la Luire

Grotte de Miramont

Aven d'Orgnac

Gouffre de Padirac

Igue de Planagrèze

Perte de Réveillon

Perte de Roc de Corn

Résurgence de Thouriès

Aven Viala

Grotte des Vipères
Causse de Cahors (Lot)

Causse Méjean (Aveyron)

Causse de Limogne (Tarn-et-Garonne)

Causse de Limogne (Tarn-et-Garonne)

Causse de Gramat (Lot)

Causse de Limogne (Tarn-et-Garonne)

Bas-Languedoc (Hérault)

Vallée du Lot (Cahors) (Lot)

Gorges de l'Hérault (Hérault)

Gorges du Verdon (Basses-Alpes)

Causse de Villeneuve (Aveyron)

Causse de Limogne (Tarn-et-Garonne)

Périgord (Dordogne)

Région Montpellièraine (Hérault)

Causse de Limogne (Tarn-et-Garonne)

Vercors (Isère)

Périgord (Dordogne)

Plateaux de l'Ardêche (Ardêche)

Causse de Gramat (Lot)

Causse de Gramat (Lot)

Causse de Gramat (Lot)

Causse de Gramat (Lot)

Gorges de l'Aveyron (Tarn-et-Gne)

Montagne Noire (Tarn)

Gorges de l'Aveyron (Tarn-et-Gne)

\section{RÉSUMÉ}

Dans cette note, qui résulte de la mise à jour d'une étude publiée en France, l'auteur définit le «système karstique», formé, au sein d'une masse calcaire, de plusieurs niveaux successifs: diaclases d'alimentation superficielles, cheminées verticales, galeries alternativement sèches et pleines d'eau selon les saisons, réseau de fentes constamment noyé.

Il étudie ensuite les modifications de ce système dues aux causes internes : corrosion, remplissage et sédimentation, concrétionnement, etc... 
Ensuite, il montre comment cette évolution du système karstique peut être modifiée par les conditions générales : géologie, tectonique, géographie, avec les pertes, les résurgences et le rôle des formations superficielles.

L'approfondissement du niveau des rivières peut créer un étagement de systèmes karstiques mais leur mise en place est toujours plus lente que le creusement et elle se produit avec du retard. En tous cas, les cavités d'un système karstique asséché subissent à leur tour une évolution qui leur est propre.

Pour terminer l'auteur définit les termes de l'évolution du système karstique: galeries embryonnaires du réseau de fentes, galeries jeunes de la zone alternativement sèche et humide, galeries mûres où le concrétionnement et la corrosion s'équilibrent, galeries vieilles où le concrétionnement devient de plus en plus important, galeries mortes où la cavité est complètement remplie par les dépôts et les concrétions.

Cette classification doit remplacer facilement les dénominations impropres de "galeries actives» et de "galeries fossiles", qui sont trop vagues et prêtent à confusion.

\section{ABSTRACT}

In this note, which has resulted from the bringing to light of a paper published in France, the author definies the "karst system" formed by several successive levels, at the heart of a limestone mass: diaclasses of surface feeding, vertical cheminies, galleries wich are alternatively dry and full of water according to the season, a hetwork of continually drowned clefts.

He then studies modifications in this system resulting from internal causes, corrosion, filling and sedimentation, concretion.

Then he shows how this evolution of the karst system may be modified by general conditions: geology, tectony, geography with the losses, résurgences and the role of surface formations.

The deepening of the river level may create a structure of differing levels in the various karst system, but their positioning is alway slowed than the streams erosion and it comes about later. In any case, the caves in a dryed karst system undergo an evolution of their own.

Finally, the author gives the definition of the terms used to explain the evolution in the karst system: "embryonic galleries" in the network of clefts, "young galleries" in the zone which is alternately wet and dry, "mature galleries" where the concretion and the erosion are balanced, "old galleries" where the concretion is becoming more and more important, "dead galleries" where the cave is completely filled by the deposits and concretions.

This classification will easely replaced the inexact terms of "active galleries" and "fossilized galleries" which are too vague and lead to confusion.

\section{BIBLIOGRAPHIE}

Toute la collection des diverses séries de Spelunca et des Annales de Spéléologie a été revue pour le présent travail. Nous avons plus spécialement utilisé les ouvrages suivants:

Balsan, L. - 1950: Grottes et abîmes des Grands Causses. Ed. Maury, Millau. Caumartin, V., et Renault, Рн. - 1958: La corrosion biochimique... Notes biospéol, t. XIII, 87-109.

Cavaillé, A. - 1936: Observations sur les phénomènes karstiques dans le Causse de Limogne. Rev. géogr. Pyrénées et $S W$ - Toulouse, t. VII, fasc. $4,392-400$. 
Cavaillé, A. - 1950: Le Bassin Karstique de la Lère. Ann. Spéléol., t. 5 (2-3), 85-98.

- 1953: L'érosion actuelle en Quercy. Res. Géomorph. Dyn., $4^{\mathrm{e}}$ année, ${ }^{\circ}{ }^{2}$, $57-75$.

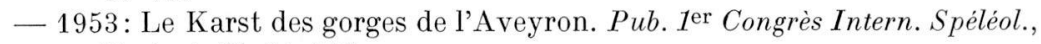
Paris, t. II, 91-103.

- 1960: Les argiles des grottes. Ann. Spéléol., t. XV (2), 383-400.

- 1961: Morphologie et Karst des Causses du Quercy. Ann. Spéléol., t. XVI, 113-131.

- 1962: C. R. III Congrès Intern. Spéléol., Vienne. Spelunca-Bull. $2^{\mathrm{e}}$ année, fasc. 1 , p. $5-23$.

- et Marty, L.-1963: Pompages et explorations en Quercy. Spelunca-Bull., $3^{\text {e }}$ année, fasc. 1, p. 7-19.

Ciry, R. - 1962: Le rôle du froid dans la spéléogénèse. 4e Congrès Nat. Spéléol., Belfort.

— 1963: Le karst de la Cote d'Or. 5e Congrès Nat. Spéléol, Millau.

Gorbel, J. - 1957: Karst du NW de l'Europe. Mêm. Rev. Géog. Lyon, 12, $541 \mathrm{p}$.

Cvijic, J. - 1918: Hydrographie souterraine et évolution morphologique du Karst. Trav. Inst. Géogr. Alp. Unio. de Grenoble, t. 6, 1-56.

Cyclopes (groupe des) - 1962: Le plateau de Presles, massif ces Coulmes. 1 vol., 184 p., Fontenay-aux-Roses.

Geze, B. - 1953: La genèse des gouffres. Actes du 1 ${ }^{\text {er }}$ Congrès Intern. Spéléol., Paris, t. II, 11-23.

- 1962: Sur quelques caractères fondamentaux des circulations karstiques. Ann. Spéléol., t. XIII, fasc. 1-4, 1958, 5-22 (importante bibliographie).

Jimenes, A. N. - 1961 : 20 años explorando a Cuba. 1 vol., 384 p., La Habana.

KUNSKY, J. - 1951: Karst et grottes, trad. Heintz. BRGM, Paris.

Licroux, R. - 1952: Le gouffre de Roque de Corn. Ann. Spéléol., t. VII, $85-90$.

Lavaur, G. de - 1950 : Le gouffre et la rivière souterraine de Padirac. Ann. Spéléol., t. V, p. 49-84.

- 1953: Hydrologie souterraine de Causse de Gramat (Lot). Pub. 1 ${ }^{\text {er }}$ Congrès Intern. Spéléol., Paris, t. II, 87-90.

Lehmann, O. - 1932: Die Hydrographie des Karstes. Enzyklop. der Erdkunde, Leipzig-Wien, 212.

Llopis-Liado - 1953: Karst holofossile et mérofossile. Pub. 1er Congrès Intern. Spéléol., Paris, 1953, 41-51.

Magné, J. et A. - 1953: Spéléologie du Causse de Labruguière (Tarn). Ann. Spéléol., t. VIII, 131-147.

Pierret, B. - 1949: Recherches souterraines dans le Département de la Dordogne. Ann. Spéléol., t. IV, 49-68.

Renault, Ph. - 1960 : Rôle de l'érosion et de la corrosion dans le creusement d'un réseau karstique. Rev. Géom. Dyn., t. XI (1), 1-4.

- 1962: Eléments de spéléomorphologie karstique, Ann. Spéléol., t. XIII, fasc. 1-4 (1958), 23-49.

Rouire, J. - 1963: Le Karst des Grands Causses. 5e Congrès Nat. Spéléol., Millau.

Seronie-Vivien, R. - 1953 : Etude des phénomènes karstiques dans l'Entredeux-Mers (Gironde). Pub. 1 er Congrès Intern. Spéléol., Paris, t. II, $75-87$. 